\title{
Consumo de medicação crónica Avaliação da prevalência no norte de Portugal
}

Cima $\mathrm{CIF}, *$ Freitas $\mathrm{RSA},{ }^{* *}$ Lamas $\mathrm{MCM},{ }^{* * *}$ Mendes $\mathrm{CASL}, * * * *$ Neves $\mathrm{AC},{ }^{* * * * *}$ Fonseca $\mathrm{C}^{* * * * * *}$

\section{RESUMO}

Introdução: É necessário conhecer a prescrição crónica, com vista à implementação de medidas para o controle, prevenção das suas complicações e optimização dos recursos disponíveis.

Objectivos: determinar a proporção do consumo de medicação crónica na população da região Norte de Portugal; caracterizar o consumo quanto ao tipo e número de medicamentos e analisar a sua associação com a idade, o sexo a situação profissional, o estado civil e o tipo de família.

Tipo de estudo: observacional analítico transversal

Local: centros de saúde pertencentes aos distritos do Porto, Braga, Viana do Castelo, Vila Real e Bragança

População: amostra aleatória de utentes com idade superior ou igual a 18 anos inscritos nas listas de 45 médicos

Métodos: Os dados foram recolhidos através de um questionário aplicado por entrevista, após convocação por postal e/ou telefone com recolha de dados por questionário.Variáveis: consumo de medicação crónica, tipo de medicamentos, número de medicamentos e diversos indicadores sócio-demográficos. Foi utilizado teste qui-quadrado e um erro a de 0,05.

Resultados: Prevalência do consumo de 58,1\% - IC95\% (56,5\%-59,8\%). Número médio de medicamentos por utente = 2,94. Os fármacos mais usados foram os do grupo Cardiovascular e Sistema Nervoso. Encontrou-se maior consumo crónico no sexo feminino, idade mais avançada, profissão (reformado e domésticas) e no tipo de família unitária. O número médio de medicamentos aumenta com a idade. Existe relação entre a idade e o consumo crónico da maioria dos grupos farmacológicos estudados.

Conclusão: Este estudo permitiu confirmar, numa maior população, os resultados existentes na literatura e reforça a necessidade de optimizar a prescrição medicamentosa.

Palavras-chave: Polifarmácia; Gestão da Terapêutica Medicamentosa; Terapêutica Farmacológica.

\section{INTRODUÇÃO}

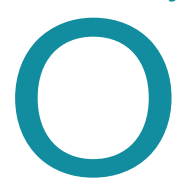

s objectivos da terapêutica medicamentosa devem ser o alívio da dor e sofrimento e a melhoria da capacidade funcional, com vista à promoção da qualidade de vida ${ }^{1} \mathrm{O}$ progressivo envelhecimento da população associado à melhoria dos cuidados de saúde tem conduzido ao aumento da prescrição e uso de medicamentos ${ }^{2,3}$.

A polimedicação é sem dúvida um grave problema

\footnotetext{
* Médica de Família na USF Ermesinde, ACES Grande Porto III-Valongo. **Médica de Família na USF Canelas, ACES Grande Porto IX-Espinho/Gaia. ***Médica de Família na USF S.Martinho, ACES Tâmega II-Vale Sousa Sul. ****Médica de Família na USF Ermesinde, ACES Grande Porto III-Valongo. *****Médica de Família na USF S.Gonçalo, ACES Tâmega I-Baixo Tâmega. ******Médica de Família na USF Garcia de Orta, ACES Grande Porto VI - Porto Ocidental.
}

que enfrenta o médico de família. Ocorre quando se utiliza um regime terapêutico com mais de cinco fármacos que são cuidadosamente seleccionados e monitorizados com um objectivo terapêutico bem defini$\mathrm{do}^{4}$. Pode ocorrer em todos os grupos etários, mas é mais frequente nos idosos pelas múltiplas co-morbilidades $^{2}$. Para além da idade, outros factores implicados na polimedicação são: multiplicidade de prescritores e falta de coordenação entre eles, automedicação e publicidade medicamentosa ${ }^{1}$.

A polimedicação contra-terapêutica é definida pela toma de cinco ou mais fármacos e um dos seguintes critérios: toma de medicamentos sem aparente indicação clínica, uso de equivalentes terapêuticos para o tratamento da mesma doença, uso simultâneo de medica- 
mentos antagonistas ou uso de outros medicamentos para tratar reacções adversas ${ }^{4}$.

Os erros de prescrição medicamentosa dizem respeito a polimedicação contra-terapêutica, uso inapropriado (prescrição de fármacos cujo potencial risco é superior ao benefício, ausência de indicação ou dose inadequada) e sub-utilização, isto é, omissão da terapêutica indicada para prevenir ou tratar determinada condição ${ }^{5} \mathrm{O}$ abuso de fármacos pode provocar numerosas reacções adversas e interacções potencialmente perigosas. As interacções medicamentosas são a principal causa de manifestações iatrogénicas em todas as faixas etárias. Esses riscos são mais elevados em pacientes hospitalizados, em especial nos idosos. Existem várias razões para uma maior incidência de iatrogenia em idosos, nomeadamente, a maior sensibilidade aos fármacos, as modificações da farmacocinética e farmacodinâmica determinadas pelo envelhecimento e a multiplicidade de patologias e fármacos utilizados ${ }^{6}$.

É cada vez mais necessário conhecer e controlar a prescrição crónica pelas suas repercussões nos gastos de saúde ${ }^{7}$. A utilização desmesurada de medicamentos, seja por automedicação, má utilização dos grupos farmacológicos, ou outras razões, leva a que esta seja uma questão importante a estudar e a debater ${ }^{8}$.

Segundo Antunes P. num estudo da sua lista de utentes, o consumo de medicação crónica é superior nas mulheres e idosos e o consumo médio de fármacos é de 2,38. Neste estudo os fármacos mais utilizados acima dos 45 anos foram os anti-hipertensores e abaixo dos 45 anos os anticonceptivos orais ${ }^{1}$. Outro estudo realizado numa população de utentes do Centro de Saúde S. João, Porto, encontrou uma prevalência de $62,7 \%$ de consumidores crónicos de fármacos, sendo também o consumo mais frequente nas mulheres e idosos. Os tipos de fármacos mais usados foram os antihipertensores $(28,2 \%)$ e os psicofármacos $(28,2 \%)^{8}$.

Em Portugal existem poucos estudos sobre a prevalência de consumo de medicação crónica na população em geral, o número e tipo de fármacos consumidos, e a sua distribuição por idade e sexo. Tendo em conta esta realidade, com os resultados deste trabalho pretendemos contribuir para o conhecimento da prevalência do consumo de fármacos, permitindo a implementação de medidas para o controle da medicação crónica e seus gastos na saúde, prevenir as suas complicações e opti- mizar o uso dos recursos disponíveis. A cronicidade da medicação foi definida pelas autoras como o uso regular por um período não inferior a 3 meses.

Os objectivos deste estudo foram: determinar a proporção do consumo de medicação crónica na população da região Norte de Portugal; caracterizar o consumo de medicação quanto ao tipo e número de medicamentos; analisar factores sócio-demográficos associados ao consumo de medicação crónica.

\section{METODOLOGIA}

Trata-se de um sub-estudo do $1 .{ }^{\circ}$ Projecto de Investigação dos Internos do Internato Médico de Medicina Geral e Familiar da Zona Norte (PRIINT1), o qual consistiu num estudo observacional analítico transversal. Decorreu entre 2005-2006 no norte de Portugal. A população do estudo correspondeu aos utentes com idade superior ou igual a 18 anos inscritos nas listas de 45 médicos de família orientadores de formação de centros de saúde pertencentes aos distritos do Porto, Braga, Viana do Castelo, Vila Real e Bragança. Foram excluídos doentes acamados, doentes com alterações cognitivas graves e grávidas. Desta população foi seleccionada informaticamente uma amostra aleatória estratificada não proporcional de 4500 indivíduos (100 utentes por cada lista de médico orientador). O cálculo da dimensão da amostra foi efectuado para uma prevalência esperada de $45 \%$, um erro alfa de 0,05 e uma precisão de 1,6\%. A dimensão total da população do estudo não foi determinada.

Foram estudadas as seguintes variáveis: idade, sexo, tipo de família, distrito residência, escolaridade, estado civil, situação profissional (estudante, empregado, desempregado e reformado), tipo de profissão e medicação.

O consumo de medicação crónica foi definido como o consumo, uma ou mais vezes por semana, por um período igual ou superior a 3 meses, seja qual for a via de administração dos fármacos pertencentes às classes terapêuticas consideradas. A classificação dos fármacos foi realizada segundo o Simposium Terapêutico. Para estudar a profissão utilizou-se a classificação adoptada pelo Instituto Nacional de Estatística em estudos populacionais, tendo sido posteriormente agrupadas para efeito de análise em profissões de colarinhos brancos e azuis.

Os dados foram recolhidos através de um questionário aplicado por entrevista, após convocação por postal 
e/ou telefone. Os entrevistadores foram os Internos do $1 .^{\circ}$ ano do Internato Médico de Medicina Geral e Familiar da Zona Norte (PRIINT1). O questionário desenvolvido pelos autores foi apresentado a todos os entrevistadores de forma a garantir a homegeneidade dos dados recolhidos e foi testado através de um teste piloto. Os entrevistados foram informados da necessidade de serem portadores de todos os medicamentos que consomem.

Os dados recolhidos foram codificados e registados em base de dados informática, com o programa Microsoft Excel, e a análise dos dados foi efectuada com SPSS $15^{\mathrm{TM}}$. Foram determinados resultados referentes à estatística descritiva e à estatística inferencial. Foi utilizado o teste do Qui-quadrado para comparação de proporções. Foi adoptado o nível de significância de 0,05.

\section{RESULTADOS}

\section{Caracterização da amostra}

A taxa de resposta foi de $77,4 \%$. Foram estudados 3481 utentes dos quais $56,1 \%$ eram do sexo feminino e $43,9 \%$ do sexo masculino. A idade variou entre os $18 \mathrm{e}$ os 97 anos, com idade média de 48,9 anos e desvio padrão de 16,7 anos. No quadro I encontra-se descrita a amostra por grupo etário, sexo e distrito.

\section{Quantidade de medicamentos consumidos}

A prevalência do consumo de medicação crónica foi de 58,1\% - IC95\% (56,5\%-59,8\%).

\begin{tabular}{|rrr|}
\hline \multicolumn{3}{|c|}{ QUADRO I. Caracterização da amostra. } \\
Grupo etário & $\mathbf{n}$ & $\%$ \\
\hline $18-25$ & 315 & 9,0 \\
$26-35$ & 575 & 16,5 \\
$36-45$ & 632 & 18,2 \\
$46-55$ & 714 & 20,5 \\
$56-65$ & 583 & 16,7 \\
$66-75$ & 460 & 13,3 \\
$>75$ & 202 & 5,8 \\
\hline Sexo & $\mathbf{n}$ & $\%$ \\
\hline Feminino & 1954 & 56,1 \\
Masculino & 1527 & 43,9 \\
\hline Distrito & $\mathbf{n}$ & $\%$ \\
\hline Braga & 585 & 16,8 \\
Bragança & 84 & 2,4 \\
Porto & 2058 & 59,1 \\
Viana & 679 & 19,5 \\
Vila Real & 75 & 2,2 \\
& &
\end{tabular}

Quanto à quantidade de medicamentos consumidos, o número variou de 1 a 15 medicamentos por utente com a média de 2,94 e o desvio-padrão de 2,05 medicamentos. Do total de utentes estudados, $30,6 \%$ consumiam apenas um medicamento - Gráfico 1.

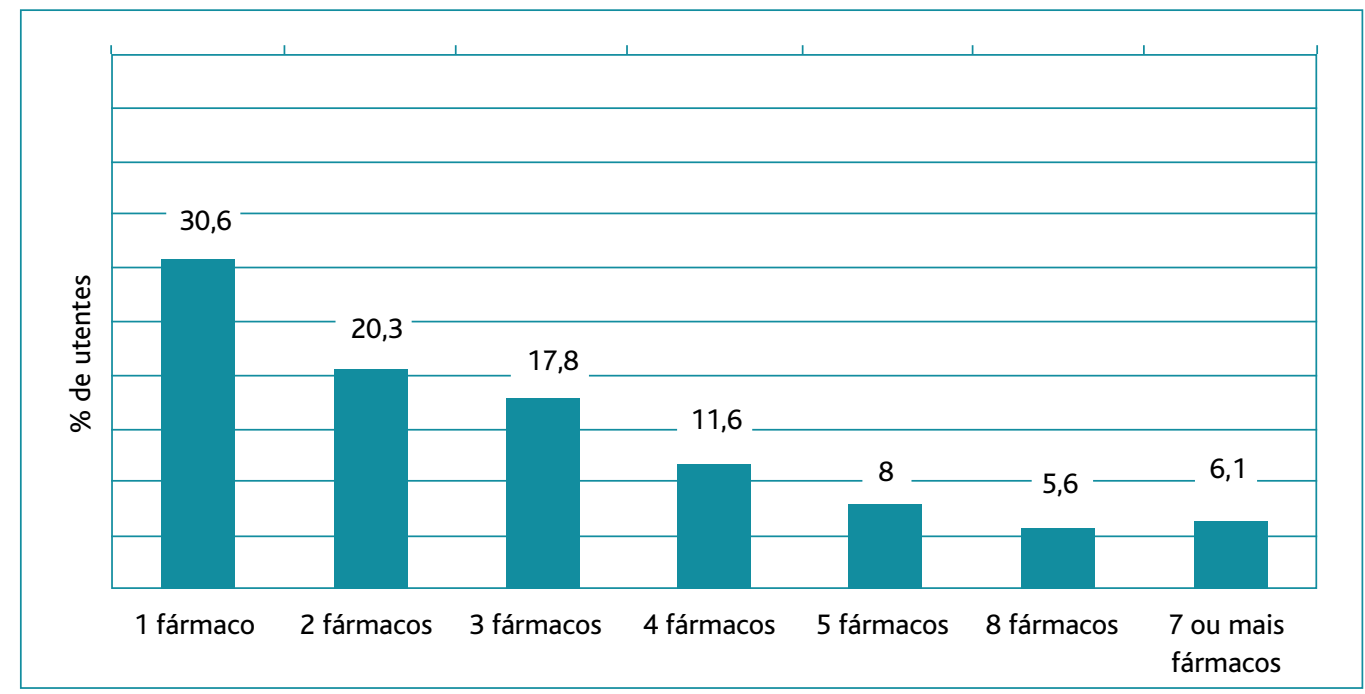

Gráfico 1. Quantidade de medicamentos consumidos por utente. 
QUADRO II. Consumo de medicação crónica por grupos de fármacos

\begin{tabular}{|lcc|} 
Grupos de fármacos & $\begin{array}{c}\text { Número de } \\
\text { utilizadores }\end{array}$ & $\%$ \\
\hline Cardiovascular & 1194 & 51,6 \\
Sistema nervoso & 887 & 38,3 \\
Metabolismo & 588 & 25,4 \\
Músculo-esquelético & 535 & 23,1 \\
Digestivo & 337 & 14,6 \\
Sangue e órgãos & 233 & 10,1 \\
hematopoiéticos & & \\
Respiratório & 169 & 7,3 \\
Genito-urinário & 130 & 5,6 \\
Fármacos hormonais & 94 & 4,1 \\
sistémicos & & \\
Terapêutica Hormonal & 86 & 3,7 \\
de Substituição & & \\
\hline
\end{tabular}

Consumo de medicação crónica por grupos de fármacos A medicação crónica mais frequentemente usada corresponde aos medicamentos do grupo cardiovascular, seguido dos fármacos do sistema nervoso, metabolismo e sistema músculo-esquelético - Quadro II.

\section{Consumo de medicação crónica por sexo, grupo etário,} distrito, escolaridade, estado civil, situação profissional, colarinhos profissionais e tipo de família

O consumo crónico de medicamentos é mais elevado no sexo feminino $(\mathrm{p}<0,001)$ e aumenta com a idade $(p<0,001)$. Verifica-se também associação estatisticamente significativa com os níveis de escolaridade mais baixos ( $<<0,001)$ e com a viuvez ( $<<0,001)$. Em relação à situação profissional, existe maior consumo no grupo dos reformados/pensionistas e das donas de casa $(\mathrm{p}<0,001)$. Não se verificaram diferenças estatisticamente significativas entre os tipos de colarinho profissional e os distritos de residência. Quanto ao tipo de família, verifica-se maior consumo as famílias unitárias e outro tipo de famílias (ex. instituições) - Quadro III e Quadro IV.

\section{DISCUSSÃO}

Neste estudo foi encontrada uma prevalência de uso de medicação crónica de 58,1\%. O consumo crónico de fármacos foi mais prevalente nas mulheres, nos idosos, nos

\begin{tabular}{|c|c|c|c|}
\hline \multicolumn{4}{|c|}{$\begin{array}{l}\text { QUADRO III. Consumo de medicação crónica por sexo, } \\
\text { grupo etário, distrito, escolaridade e estado civil }\end{array}$} \\
\hline & \multicolumn{2}{|c|}{ Medicação crónica } & \multirow{2}{*}{ P* } \\
\hline & $\mathrm{n}$ & $\%$ & \\
\hline \multicolumn{4}{|l|}{ Sexo } \\
\hline Feminino & 1954 & 63,8 & $P<0,001$ \\
\hline Masculino & 1527 & 50,9 & \\
\hline \multicolumn{4}{|l|}{ Grupo etário } \\
\hline $18-25$ & 315 & 12,7 & \\
\hline $26-35$ & 575 & 26,6 & \\
\hline $36-45$ & 632 & 43,5 & \\
\hline $46-55$ & 714 & 67,8 & $P<0,001$ \\
\hline $56-65$ & 583 & 80,3 & \\
\hline $66-75$ & 460 & 91,3 & \\
\hline$>75$ & 202 & 91,1 & \\
\hline \multicolumn{4}{|l|}{ Distrito } \\
\hline Braga & 585 & 55,2 & \\
\hline Porto & 2058 & 59,2 & $P=0,14$ \\
\hline Viana & 679 & 58,8 & \\
\hline Vila Real + Bragança & 159 & 52,2 & \\
\hline \multicolumn{4}{|l|}{ Escolaridade } \\
\hline Analfabetos & 192 & 80,2 & $P<0,001$ \\
\hline 1. ${ }^{\mathrm{a}}-4 .^{\mathrm{a}}$ classe & 1372 & 75,3 & \\
\hline $5 .^{\circ}-9 .^{\circ}$ ano & 909 & 47,9 & \\
\hline $10 .^{\circ}-12 .^{\circ}$ ano & 553 & 38,9 & \\
\hline Curso superior ou mais & 410 & 38,0 & \\
\hline \multicolumn{4}{|l|}{ Estado civil } \\
\hline Solteiro & 608 & 28,3 & $P<0,001$ \\
\hline Casado/União de facto & 2470 & 62,0 & \\
\hline Viúvo & 259 & 90,0 & \\
\hline Separado/Divorciado & 140 & 61,4 & \\
\hline
\end{tabular}

analfabetos, nos viúvos e nos membros de famílias unitárias, nos reformados/pensionistas e nas donas de casa. Os tipos de fármacos mais utilizados foram os medicamentos do grupo cardiovascular, sistema nervoso, metabolismo e musculo-esquelético. Na população estudada, 19,7\% eram consumidores de 5 ou mais medicamentos.

A prevalência de uso de medicação crónica no presente estudo foi superior à encontrada em estudos anteriores. Num estudo realizado na área de Lisboa em 1994 foi encontrada uma prevalência de uso crónico de fármacos de $39 \%$ em utentes inscritos numa lista de um médico de família ${ }^{1}$. Em outro estudo realizado em 


\begin{tabular}{|c|c|c|c|}
\hline \multicolumn{4}{|c|}{$\begin{array}{l}\text { QUADRO IV. Consumo de medicação crónica por } \\
\text { situação profissional, colarinhos profissionais e tipo de } \\
\text { família }\end{array}$} \\
\hline & \multicolumn{2}{|c|}{ Medicação crónica } & \multirow{2}{*}{ P* } \\
\hline & $\mathbf{n}$ & $\%$ & \\
\hline \multicolumn{4}{|l|}{ Situação Profissional } \\
\hline Estudante & 124 & 15,3 & \multirow{6}{*}{$P<0,001$} \\
\hline Dona de casa & 318 & 74,2 & \\
\hline Reformado/Pensionista & 922 & 88,3 & \\
\hline Trabalhador activo & 1787 & 43,6 & \\
\hline Desempregado & 266 & 58,3 & \\
\hline Trabalhador-estudante & 44 & 18,2 & \\
\hline \multicolumn{4}{|l|}{ Colarinhos } \\
\hline Azul & 627 & 42,1 & \multirow{2}{*}{$P=0,095$} \\
\hline Branco & 1463 & 46,1 & \\
\hline \multicolumn{4}{|l|}{ Tipo de Família } \\
\hline Unitária & 276 & 69,6 & \multirow{6}{*}{$P<0,001$} \\
\hline Nuclear & 2546 & 55,0 & \\
\hline Alargada & 375 & 65,1 & \\
\hline Monoparental & 137 & 63,5 & \\
\hline Reconstruída & 49 & 65,3 & \\
\hline Outro tipo & 78 & 69,2 & \\
\hline
\end{tabular}

* Teste do qui-quadrado

2002/03, também na área de Lisboa, com base na população de utentes de dois centros de saúde, foi encontrada uma prevalência de polimedicação de $48 \%{ }^{17}$. Num estudo realizado em 2004 no Centro de Saúde S. João no Porto numa lista de utentes foi encontrada uma prevalência de $41,5 \%$.

O consumo crónico de medicamentos e a polimedicação foram mais prevalentes nos idosos, um resultado esperado dado que este grupo etário apresenta maior comorbilidade, o que coincide com outros estudos realizados anteriormente.

O maior consumo por parte das mulheres também foi encontrado nos três estudos realizados em Portugal, referidos anteriormente. Nos restantes estudos poderá ser parcialmente justificado pelo uso de anticoncepcionais orais. No presente estudo foi excluído o consumo destes, pelo que estas prevalências podem estar relacionadas com o facto das mulheres serem mais consumidoras dos serviços de saúde.

Foi encontrada relação entre o tipo de família e o con- sumo crónico de fármacos, sendo este mais prevalente nos membros de famílias unitárias. As famílias unitárias são muitas vezes constituídas por viúvos e idosos o que pode induzir um efeito de confundimento com a idade e o sexo. No estudo, anteriormente referido, realizado na área de Lisboa em 1994, as famílias unitárias foram as que apresentaram os valores mais baixos de consumo de fármacos e as famílias nucleares valores mais elevados ${ }^{1}$. Estas diferenças podem ser explicadas pela variação no tipo de famílias da nossa sociedade entre 1994 e 2005. Não foi encontrada relação entre o tipo de família e o consumo crónico de fármacos, o seu número ou tipo, no estudo realizado em 2004 no Centro de Saúde S. João ${ }^{9}$. Não pode, contudo, ser excluído um viés de informação nestes estudos, dado que os dados constantes nos processos clínicos poderão não estar actualizados.

Em relação à situação profissional, existe maior consumo no grupo dos reformados/pensionistas e no grupo das donas de casa, mas também neste caso poderá existir efeito confundidor com a idade e o sexo. Quando estudados apenas os trabalhadores activos, não encontrámos qualquer associação entre o consumo de medicação crónica e o tipo de profissão (colarinhos).

Apesar da classificação da situação profissional utilizada no estudo realizado em 2003 em Lisboa ser a mesma utilizada neste estudo, apenas foi utilizada para caracterizar a amostra e não foi analisada a possível associação com o consumo de fármacos ${ }^{17}$. No estudo realizado no Centro de Saúde S. João no Porto em 2004 foi encontrado um maior consumo crónico nos reformados, sendo que estas diferenças de consumo se revelaram independentes da idade ${ }^{9}$.

Consideramos como aspectos positivos deste estudo o facto de a dimensão da amostra ser largamente superior às representadas nos estudos realizados até à data, e de a amostra ser constituída por elementos de várias listas e dos diferentes distritos da região norte. A taxa de resposta foi boa, sendo que este resultado se deveu provavelmente à informação prévia por postal e/ou telefone da realização da entrevista e da necessidade dos entrevistados serem portadores das embalagens de fármacos que utilizavam. $\mathrm{O}$ facto de a amostragem ter sido efectuada por aleatorização de todos os inscritos e não apenas dos utilizadores também contribui para minimizar o viés de selecção.

$\mathrm{O}$ facto de a colheita de dados se ter baseado num 
questionário aplicado por entrevista e não apenas por consulta do processo clínico dos utentes tentou minimizar um possível viés de informação. Importa, também, referir que o Médico de Família não é o único prescritor, podendo levar, portanto, a uma prevalência subestimada do uso crónico de medicamentos nas amostras anteriormente estudadas. Existem também vários medicamentos que não são de prescrição médica obrigatória, podendo o seu uso crónico passar despercebido ao Médico de Família. Neste estudo tentou-se minimizar este viés pedindo ao utente para se fazer acompanhar da sua medicação habitual e não apenas da prescrita.

É de salientar a escassez de estudos sobre o consumo de medicação crónica em adultos não idosos, pelo que este estudo se torna oportuno para melhor conhecimento do uso crónico de fármacos na população adulta.

Em face dos resultados encontrados, é oportuno procurar sensibilizar os Médicos de Família para o conhecimento dos hábitos medicamentosos dos seusutentes. Este conhecimento poderá ser adquirido através do contacto individualizado médico-utente e, de uma forma mais ampla, através de estudos que permitam aprofundar os conhecimentos sobre o consumo crónico de medicamentos.

Os fármacos mais utilizados pelos utentes como medicação crónica são os anti-hipertensores, os antidislipidémicos e os anticoagulantes/antitrombóticos, reflectindo a elevada prevalência de factores de risco e de doença cardiovascular na população. Importa, também nesta área, apostar na promoção de um estilo de vida saudável que ajude a prevenir e/ou a tratar estas patologias, evitando uma menor recorrência a fármacos.

Particularmente no caso dos psicofármacos, cuja prevalência de uso crónico foi elevada neste estudo, importa ter em conta a formação dos médicos e a educação dos utentes na tentativa de explorar terapêuticas não medicamentosas, que permitam a redução do consumo destes fármacos.

Uma área que se tem demonstrado com crescente interesse é o estudo da qualidade de prescrição (por exemplo, estudos de indicação-prescrição, estudos de prescrições potencialmente inapropriadas). A realização de estudos nesta área permitirá não só o conhecimento dos hábitos medicamentosos da população mas também a adequação desses hábitos, podendo servir de ponto de partida para medidas que promovam um uso mais racional dos medicamentos.
$\mathrm{Na}$ abordagem de cuidados preventivos deve ser dada ênfase às intervenções não farmacológicas sempre que possível antes de iniciar uma medicação. Os Médicos de Família necessitam de tempo e recursos adequados para a revisão da medicação nos doentes polimedicados, especialmente nos idosos. As revisões terapêuticas devem ser efectuadas pelo menos uma vez por ano. Os cartões de medicação crónica podem melhorar a segurança da prescrição.

A utilização de sistemas tecnológicos de informação diminui os erros de prescrição e devem ser usados sempre que possível para apoiar a prescrição. A maioria dos estudos existentes aponta para uma redução drástica nos erros de medicação relacionados com a prescrição após implementação da prescrição por computador (estimada por diferentes autores entre 66 a 94\%, aliada a um suporte clínico de decisão). Outros tipos de erros encontrados incluem ainda erros de na via de administração, troca na identificação de doentes e discrepâncias na prescrição ${ }^{20}$.

É necessária melhor comunicação entre os Cuidados de Saúde Primários (CSP) e os Cuidados de Saúde Secundários (CSS) para uma correcta actualização da terapêutica instituída ou modificada. Deve estar disponível informação independente e baseada na evidência sobre a terapêutica que deverá ser regularmente actualizada e revista conjuntamente pelos profissionais dos CSP e CSS. Os medicamentos genéricos devem ser claramente identificados para melhorar a adesão e evitar erros na sua toma.

Os pacientes, por sua vez, têm a responsabilidade de serem conhecedores dos seus medicamentos incluindo os medicamentos não sujeitos a receita médica. Nos doentes polimedicados, e principalmente nos idosos, há uma maior probabilidade de erros de prescrição e de toma dos medicamentos, aumentando desta forma o risco de reacções adversas.

Devem ser tomadas medidas de forma a assegurar a formação médica contínua e a promoção do ensino dos doentes, com o objectivo final de uma prescrição segura e eficiente.

\section{AGRADECIMENTOS}

Aos Médicos Internos de Medicina Geral e Familiar e seus Orientadores de Formação envolvidos na recolha de dados/elaboração do estudo, bem como aos restantes elementos das equipas das unidades de saúde envolvidas, administrativos e enfermeiros. 


\section{REFERÊNCIAS BIBLIOGRÁFICAS}

1. Antunes P. Medicação crónica numa lista de utentes. Rev Port Clin Geral 2000 Mar-Abr; 16 (2): 113-24.

2. Couto L, Rio C. Polimedicação no Idoso. Rev O Médico 1991; 96: 11-6.

3. Esteves MJS, Azevedo L, Couto L. Polimedicação crónica major. Rev Med Univ 2005; 49-56.

4. Wick JY, Zanni GR. Geriatric Pharmacology. Nov 2004. Disponível em http://www.pharmacytimes.com [acedido em 27/10/2011].

5. American Medical Association Council. Improving the quality of geriatric pharmacotherapy: Report 5 of the 2002 AMA Annual Meeting. Disponível em: http://www.ama-assn.org/ama/no-index/aboutama/13592.shtml [acedido em 27/02/2011].

6. Ballone, GB. O uso de medicamentos em idosos e iatrogenia. Disponível em http://sites.uol.com.br/gballone/geriat/medicam.html [acedido em 27/02/2011].

7. Díaz Barroso A, Albaladejo Blanco C, Girona Amores A, Ancochea Serraïma L, Borrego Crespo R. Estudio descriptivo de la medicación crónica autorizada en el ABS de Llefiá (Badalona). Aten Primária 1996 Mar $15 ; 17(4): 251-6$

8. Martinez PH, Polo AC. Estúdio del consumo de fármacos en la Residencia del IMSERSO. Rev Esp Geriatr Gerontol 2002; 37 (3): 134-40.

9. Ferreira R. Consumo crónico de medicamentos na população de um Centro de Saúde. Rev Port Clin Geral 2007 Mar-Abr; 23 (2): 125-32.

10. Tomàs MT, Centelles F, Valero C, Alcalá A, Cerón A, Soler J, et al. Prescripción crónica de fármacos en pacientes geriátricos de un centro de salud urbano. Aten Primaria 1999 Feb 28; 23 (3): 121-6.

11. Silva INT, Lindolpho MC, Dutra PAP, Sá SPC. O enfermeiro e o paciente idoso em terapêutica plurimedicamentosa. Rev UFG 2003; 5 (2). FDisponível em: http://www.proec.ufg.br [acedido em 27/02/2011].

12. Stafford RS, Davidson SM, Davidson H, Miracle-McMahill H, Crawfford $S L$, Blumenthal D. Chronic disease medication use in managed care and indemnity insurance plans. Health Serv Res 2003 Apr; 38 (2): 595-612.
13. Fick DM, Cooper JW, Wade WE, Waller JL, Maclean R, Beers MH. Updating the Beers criteria for potencially inappropriate medication use in older adults: results of a consensus panel of experts. Arch Intern Med 2003 Dec 8-22; 163 (22): 2716-24.

14. Avorn J. Improving drug use in erdely patients: getting to the next level. JAMA 2001 Dec 12; 286 (22): 2866-8.

15. Morley JE. Drugs, aging and the future. J Gerontol A Biol Sci Med Sci 2002 Jan; 57(1): M2-6

16. Broeiro P, Ramos V. Patologia múltipla e polifarmácia no idoso. Rev Port Clin Geral 1997 Jan-Fev; 14 (1): 8-22.

17. Silva P, Luís S, Biscaia A. Polimedicação: um estudo de prevalência nosCentros de Saúde do Lumiar e de Queluz. Rev Port Clin Geral 2004 Mai-Jun; 20 (3): 323-36.

18. Sousa M. Perfil dos utilizadores de psicofármacos na Unidade de Saúde Familiar de Canelas. Rev Port Clin Geral 2007 Jan-Fev; 23 (1): 33-42.

19. Recomendação da UEMO para uma prática medicamentosa mais segura no idoso. Rev Port Clin Geral 2006 Jan-Fev; 22 (1): 111-2.

20. Tipos de erros de medicação descritos. Infarmed - Boletim de Fármaco-Vigilância 2007; 11 (3).

\section{CONFLITOS DE INTERESSE}

Os autores declaram não existirem conflitos de interesse na elaboração deste artigo.

\section{ENDEREÇO PARA CORRESPONDÊNCIA \\ Catarina Cima \\ Rua 9 de Abril, n. 149 - 3. ${ }^{\circ}$ direito frente \\ 4250-347 Porto \\ e-mail: catcima@portugalmail.com}

Recebido em 11/12/2007

Aceite para publicação em 17/12/2010

\section{ABSTRACT}

\section{USE OF CHRONIC MEDICATION. PREVALENCE IN NORTHERN PORTUGAL}

Introduction: It is necessary to know the chronic prescription, with a view towards the implement of measures to control, to prevent their complications and to optimise the available resources. The aims of that study were: to determine the proportion of adult patients chronically-medicated in the north of Portugal; to characterise the consumption, as far as the type and number of medicines are concerned, and to analyse their association with age, gender, professional situation, marital status and type of family.

Methodology: Transversal analytical study with gather of data through questionnaires. Random sample of 45 doctors' patients of health centres from the north of Portugal. Variables studied were consumption of chronicle medication, type of medicines, number of medicines, demographic variations. Used test chi-squared, $a=0,05$.

Results: Consumption's prevalence $=58,1 \%$. Average number of medicines per patient $=2,94$. The most used medicines were those from the cardiovascular and nervous system groups. There was found an association between chronicle consumption and females, elder ages, jobs (retired or housekeepers) and single-element families. The average number of medicine rises with the age. There is a relation between the age and most of the studied pharmacological groups.

Discussion: This study allowed confirming, in a bigger population, the results in literature and reinforces the necessity of optimising the prescription of medicines.

Keywords: Polypharmacy; Medication Therapy Management; Drug Therapy. 


\section{ANEXO I \\ QUESTIONÁRIO}

Questionário

N. ${ }^{\circ}$

$P_{1}$. Data de nascimento:

_L_ (_) anos

$P_{2}$. Sexo:

0[]$M$

1[] $\mathrm{F}$

$\mathbf{P}_{3}$. Consome algum medicamento há pelo menos 3 meses?
( $\geq 3$ meses)
0 [ ] Não
1 [ ] Sim
9 [ ] Não sabe

$\mathbf{P}_{4}$. Quantos medicamentos consome há pelo menos 3 meses?

9 [ ] não aplicável

De que grupo(s) farmacológico(s)?

$\mathbf{P}_{5}$. Aparelho digestivo?

${ }_{1}$ [ ] sim ${ }_{0}$ [ ] não ${ }_{9}$ [ ] não aplicável

$\mathbf{P}_{6}$. Metabolismo?

${ }_{1}$ [ ] sim ${ }_{0}$ [ ] não ${ }_{9}$ [ ] não aplicável

$\mathbf{P}_{7}$. Aparelho cardiovascular?

${ }_{1}$ [ ] sim ${ }_{0}$ [ ] não ${ }_{9}$ [ ] não aplicável

$\mathbf{P}_{8}$. Sangue e órgãos hematopoiéticos?

${ }_{1}$ [ ] sim ${ }_{0}$ [ ] não ${ }_{9}$ [ ] não aplicável

$P_{9}$. Aparelho respiratório?

${ }_{1}$ [ ] sim ${ }_{0}$ [ ] não ${ }_{9}$ [ ] não aplicável

$P_{10}$. Aparelho génito-urinário?

${ }_{1}$ [ ] sim ${ }_{0}$ [ ] não ${ }_{9}$ [ ] não aplicável

$P_{11}$. Hormonas sexuais?

${ }_{1}$ [ ] sim ${ }_{0}$ [ ] não ${ }_{9}$ [ ] não aplicável

$\mathbf{P}_{12}$. Sistema músculo-esquelético?

${ }_{1}$ [ ] sim ${ }_{0}$ [ ] não ${ }_{9}$ [ ] não aplicável

$\mathbf{P}_{13}$. Sistema nervoso?

${ }_{1}$ [ ] sim ${ }_{0}$ [ ] não ${ }_{9}$ [ ] não aplicável

$\mathbf{P}_{14}$. Fármacos hormonais sistémicos?

${ }_{1}$ [ ] sim ${ }_{0}$ [ ] não ${ }_{9}$ [ ] não aplicável

$\mathbf{P}_{15}$. Outros?

${ }_{1}$ [ ] sim ${ }_{0}\left[\right.$ [ ] não ${ }_{9}$ [ ] não aplicável

Instruções de preenchimento do questionário

- Se em P3 respondeu Não ou Não sabe, deve assinalar uma cruz em não aplicável nas perguntas $\mathrm{P}_{4}$ a $\mathrm{P}_{15}$.

- A tabela seguinte deverá servir de apoio ao preenchimento correcto das respostas às perguntas $\mathrm{P}_{5}$ a $\mathrm{P}_{15}$. Caso surjam dúvidas na classificação dos fármacos, deverá ser consultado o Simposium Terapêutico ${ }^{\circledR}$ e usada a sua classificação.

\begin{tabular}{|c|c|}
\hline Aparelho digestivo & $\begin{array}{l}\text { Anti-ácidos, anti-ulcerosos } \\
\text { Laxantes } \\
\text { Outros }\end{array}$ \\
\hline Metabolismo & $\begin{array}{l}\text { Antidiabéticos (orais e insulina) } \\
\text { Vitaminas e suplementos minerais } \\
\text { Outros }\end{array}$ \\
\hline $\begin{array}{l}\text { Aparelho } \\
\text { cardiovascular }\end{array}$ & $\begin{array}{l}\text { IECA, ARA II } \\
\text { Antagonistas do } \mathrm{Ca}^{2+} \\
\text { Diuréticos } \\
\text { Bloqueadores beta } \\
\text { Digitálicos, anti-disrítmicos, nitratos } \\
\text { Vasoprotectores } \\
\text { (antivaricosos, estabilizadores capilares) } \\
\text { Antidislipidémicos } \\
\text { Outros }\end{array}$ \\
\hline $\begin{array}{l}\text { Sangue e órgãos } \\
\text { hematopoiéticos }\end{array}$ & $\begin{array}{l}\text { Antitrombóticos } \\
\text { (anticoagulantes e anti-agregantes } \\
\text { plaquetários) } \\
\text { Anti-anémicos } \\
\text { Outros }\end{array}$ \\
\hline Aparelho respiratório & $\begin{array}{l}\text { Fármacos para doenças obstrutivas das vias } \\
\text { aéreas } \\
\text { Anti-histamínicos } \\
\text { Outros }\end{array}$ \\
\hline $\begin{array}{l}\text { Aparelho génito- } \\
\text {-urinário }\end{array}$ & $\begin{array}{l}\text { Anti-espasmódicos } \\
\text { Fármacos usados na hipertrofia benigna da } \\
\text { próstata e na disfunção eréctil } \\
\text { Outros }\end{array}$ \\
\hline Hormonas sexuais & $\begin{array}{l}\text { Hormonas sexuais } \\
\text { Outros }\end{array}$ \\
\hline $\begin{array}{l}\text { Sistema músculo- } \\
\text {-esquelético }\end{array}$ & $\begin{array}{l}\text { Anti-inflamatórios, anti-reumáticos } \\
\text { Antigotosos } \\
\text { Bifosfonatos } \\
\text { Outros }\end{array}$ \\
\hline Sistema nervoso & $\begin{array}{l}\text { Analgésicos } \\
\text { Psicolépticos (anti-psicóticos, ansiolíticos, } \\
\text { sedativos, hipnóticos) } \\
\text { Psicoanalépticos (antidepressivos, } \\
\text { psicoestimulantes, fármacos anti-demência) } \\
\text { Anti-epilépticos } \\
\text { Anti-parkinsónicos } \\
\text { Anti-vertiginosos } \\
\text { Outros }\end{array}$ \\
\hline $\begin{array}{l}\text { Fármacos hormonais } \\
\text { sistémicos }\end{array}$ & $\begin{array}{l}\text { Corticóides } \\
\text { Terapêutica tiroideia } \\
\text { Outros }\end{array}$ \\
\hline
\end{tabular}

